\title{
Mini-Review: Hydrogen Atoms in a High-Frequency Laser Field
}

\section{Eugene Oks}

Physics Department, 380 Duncan Drive, Auburn University, Auburn, AL 36849, USA; goks@physics.auburn.edu

Received: 9 July 2019; Accepted: 15 August 2019; Published: 19 August 2019

\begin{abstract}
Because of the continuing advances in developing lasers in the far-ultraviolet and x-ray ranges, studies of the behavior of atoms under a high-frequency laser field are of theoretical and practical interest. In the present paper, we review various analytical results obtained by the method of separating rapid and slow subsystems for various polarizations of the laser field. Specifically, we review the corresponding analytical results both in terms of the quantum description of the phenomena involved and in terms of the classical description of the phenomena involved. We point out that, for the classical description of hydrogen atoms in a high-frequency laser field, there are interesting celestial analogies. We discuss hidden symmetries of these physical systems, the advantages of this analytical method, and the connection between these results and the transition to chaos.
\end{abstract}

Keywords: atoms in a high-frequency laser field; separation of rapid and slow subsystems; celestial analogies; hidden symmetries

\section{Introduction}

Because of the continuing advances in developing lasers in the far-ultraviolet and x-ray ranges, studies of the behavior of atoms under a high-frequency laser field are of theoretical and practical interest. The "high-frequency" means that the laser frequency $\omega$ is much greater than any of the atomic transition frequencies $\omega_{n^{\prime} n}$ :

$$
\omega>\omega_{\text {n'n }}
$$

In the present paper we review various analytical results obtained by the method of separating rapid and slow subsystems. Specifically, in Section 2 we review the corresponding analytical results in frames of the quantum description of the phenomena involved. In Section 2 we follow papers [1,2]. In Section 3 we review the corresponding analytical results in frames of the classical description of the phenomena involved. In Section 3 we follow papers [3,4]. In Conclusions, we discuss the advantages of applying the method of separating rapid and slow subsystems to the physical systems under consideration. We emphasize the rich physics behind the corresponding phenomena.

\section{Quantum Hydrogen Atoms in a High-Frequency Laser Field}

It is well-known [5,6] that, for quantum systems in a monochromatic field, it is convenient to use the formalism of quasienergy states. The problem of finding such states of the hydrogen-like atom/ion in a linearly polarized high-frequency laser field was first considered by Ritus [5]. He found quasienergies for the states of the principal quantum number $n=1$ and $n=2$, for which the perturbation operator $U$ is diagonal in the basis of the spherical wave functions (i.e., the wave functions of the unperturbed atom in the spherical quantization).

Papers $[7,8]$ stated, without any proof, that for states of $n>2$, the perturbation operator U couples the substates of $l$ and $l \pm 2$, where $l$ is the orbital momentum quantum number. In paper [8], the study of the quasienergies for $n>2$ was based on the approximate analogy with the problem of finding 
energies for the hydrogen molecular ion $\mathrm{H}_{2}{ }^{+}$. Based on the energies for $\mathrm{H}_{2}{ }^{+}$from paper [9], for $\mathrm{n}>2$, the author of paper [8] found "corrections" to the quasienergies from paper [5] due to the coupling of the substates of $l$ and $l \pm 2$. However, in a later paper [10], where the authors used the dependence of the energies for $\mathrm{H}_{2}{ }^{+}$on the internuclear distance $\mathrm{R}$ from paper [11], they obtained the result that in the limit $\mathrm{R} \rightarrow \infty$ coincides with the quasienergies from paper [5], which contradicts paper [8].

Thus, it remained unclear whether the perturbation $\mathrm{U}$ couples the substates of $l$ and $l \pm 2$ at fixed $n$. To answer this question, one should have directly calculated matrix elements of the perturbation between the substates of $l$ and $l \pm 2$ at fixed $\mathrm{n}$. This was accomplished in paper [1], where the authors demonstrated that these matrix elements are zeros. This meant that, in reality, the perturbation operator $\mathrm{U}$ does not couple the substates of $l$ and $l \pm 2$ for any $\mathrm{n}$, so that the expression for quasienergies from paper [5] is not limited by $n=1$ and $n=2$ (as asserted in paper [5]), but is in fact valid for any $n$. The diagonality of the perturbation operator $U$ in the basis of the spherical wave functions allowed easily calculating the splitting of any spectral line of a hydrogenic atom/ion under the high-frequency laser field. This was the main, fundamental result of paper [1].

Under condition (1), the laser field represents the rapid subsystem, while the hydrogenic atom/ion represents a slow subsystem. The powerful method of separating rapid and slow subsystems allows us to obtain accurate analytical results for such systems. For implementing this method for particular physical systems, there can be some interesting nuances or versions. It is instructive to see how this method was implemented in paper [1], whose results we present below.

The Schrödinger equation for ahydrogen-like atom/ion in a laser field (of the amplitude $\mathrm{E}_{0}$ ) described by the vector-potential $\mathbf{A}(\mathrm{t})=\mathbf{A}_{0} \sin \omega \mathrm{t}$, where $\mathbf{A}_{0}=\left(0,0,-\mathrm{cE}_{0} / \omega\right)$, has the following form (here and below the atomic units $\hbar=\mathrm{m}_{\mathrm{e}}=\mathrm{e}=1$ are used):

$$
\mathrm{i} \partial \Psi / \partial \mathrm{t}=\left[\mathrm{H}_{0}+\mathrm{V}(\mathrm{t})\right] \Psi, \mathrm{H}_{0}=\mathbf{p}^{2} / 2-\mathrm{Z} / \mathrm{r}+\mathrm{A}_{0}^{2} /(2 \mathrm{c}), \mathrm{V}(\mathrm{t})=-\left(\mathbf{A}_{0} \mathbf{p} / \mathrm{c}\right) \sin \omega \mathrm{t}-\left[\mathrm{A}_{0}^{2} /(2 \mathrm{c})\right] \cos 2 \omega \mathrm{t}
$$

Here, $Z$ is the nuclear charge and $r$ is the distance of the electron from the nucleus. The notation $\mathbf{A}_{0} \mathbf{p}$ stands for the scalar product (also known as the dot-product) of these two vectors. The term $\mathrm{A}_{0}^{2} /(2 \mathrm{c})$ is the average vibrational energy of the free electron in the laser field.

We seek the solution of Equation (2) in the form

$$
\Psi(\mathrm{t})=\exp [-\mathrm{i} \alpha(\mathrm{t})] \Phi, \alpha(\mathrm{t})=\left[\mathbf{A}_{0} \mathbf{p} /(\omega \mathrm{c})\right] \cos \omega \mathrm{t}-\left[\mathrm{A}_{0}^{2} /\left(8 \omega \mathrm{c}^{2}\right)\right] \sin 2 \omega \mathrm{t}
$$

Substituting Equation (3) in Equation (2), we obtain the following equation:

$$
\mathrm{i} \partial \Phi / \partial \mathrm{t}=\mathrm{H}_{1} \Phi, \mathrm{H}_{1}=\exp [\mathrm{i} \alpha(\mathrm{t})] \mathrm{H}_{0} \exp [-\mathrm{i} \alpha(\mathrm{t})]=\mathrm{H}_{0}+\mathrm{i}\left[\alpha, \mathrm{H}_{0}\right]+\left(\mathrm{i}^{2} / 2\right)\left[\alpha,\left[\alpha, \mathrm{H}_{0}\right]\right]+\ldots=\mathrm{H}_{1, \text { stat }}+\mathrm{H}_{1, \text { osc }}
$$

In Equation (4), $\left[\alpha, \mathrm{H}_{0}\right]$ and $\left[\alpha,\left[\alpha, \mathrm{H}_{0}\right]\right]$ are commutators. $\mathrm{H}_{1, \text { stat }}$ and $\mathrm{H}_{1, \mathrm{osc}}$ are the stationary (i.e., averaged over the laser field period $2 \pi / \omega$ ) and oscillatory parts of the Hamiltonian $\mathrm{H}_{1}$. Under the condition (1), i.e., since the laser field is the rapid subsystem, the primary contribution to the solution of Equation (4) originates from the stationary part $\mathrm{H}_{1, \text { stat }}$. According to the second line of Equation (4), $\mathrm{H}_{1, \text { stat }}$ can be represented in the following form:

$$
\mathrm{H}_{1, \text { stat }}=\mathrm{H}_{0}+\gamma \mathrm{V}_{1}+\gamma^{2} \mathrm{~V}_{2}+\ldots, \gamma=\mathrm{E}_{0}^{2} /\left(2 \omega^{2}\right)^{2}, \mathrm{~V}_{1}=\mathrm{Z}\left(1-3 \cos ^{2} \theta\right) / \mathrm{r}^{3}, \mathrm{~V}_{2}=3 \mathrm{Z}\left(-3+30 \cos ^{2} \theta-35 \cos ^{4} \theta\right) /\left(4 \mathrm{r}^{5}\right)
$$

where $\theta$ is the polar angle of the atomic electron, the z-axis being parallel to the laser field.

Assuming $\gamma<<1$, we will use the perturbation theory for finding the eigenvalues and the eigenfunctions of the Hamiltonian $\mathrm{H}_{1 \text {,stat. }}$. It is important to emphasize the following counterintuitive fact: since the unperturbed system is degenerate, then according to paper [12], the linear (with respect to $\gamma$ ) corrections to the eigenfunctions will originate not only from the term $\gamma \mathrm{V}_{1}$ but also from the term $\gamma^{2} \mathrm{~V}_{2}$. 
It is easy to see that the radial part of the matrix element $\left\langle\mathrm{n} l \mathrm{~m}\left|\mathrm{~V}_{1}\right| \mathrm{n} l^{\prime} \mathrm{m}\right\rangle$, where $l^{\prime}=l-2$, reduces to the following type of the integral:

$$
J=\int_{0}^{\infty} z^{r} \exp (-z) Q_{k+s}^{r}(z) Q_{k}{ }^{p}(z) d z,(s>0),
$$

where $\mathrm{Q}_{\mathrm{n}}{ }^{\mathrm{m}}(\mathrm{z})$ are the Laguerre polynomials. According to the textbook [13], for $<\mathrm{n} l \mathrm{~m}\left|\mathrm{~V}_{1}\right| \mathrm{n} l^{\prime} \mathrm{m}>$ with $l^{\prime}=l-2$ one gets $\mathrm{J}=0$, so that $\left\langle\mathrm{n} l \mathrm{~m}\left|\mathrm{~V}_{1}\right| \mathrm{n} l^{\prime} \mathrm{m}>=0\right.$. This means that the spherical eigenfunctions $\varphi_{\mathrm{n} l m}$ of the unperturbed Hamiltonian $\mathrm{H}_{0}$ turn out to be the correct eigenfunctions of the zeroth order of the truncated perturbed Hamiltonian $\mathrm{H}_{0}+\gamma \mathrm{V}_{1}$. Therefore, according to paper [12], the eigenvalues $\mathrm{F}_{\mathrm{n} \lambda}$ and the eigenfunctions $\chi_{\mathrm{n} \lambda}$ of the Hamiltonian $\mathrm{H}_{1 \text {,stat }}$ within the accuracy of the terms $\sim \gamma$ are expressed as follows:

$$
\begin{gathered}
\mathrm{F}_{\mathrm{n} \lambda}=\mathrm{E}_{\mathrm{n}}{ }^{(0)}+\gamma<\mathrm{n} \lambda\left|\mathrm{V}_{1}\right| \mathrm{n} \lambda>, \quad \mathrm{E}_{\mathrm{n}}{ }^{(0)}=-\mathrm{Z}^{2} /\left(2 \mathrm{n}^{2}\right)+\mathrm{E}_{0}{ }^{2} /(2 \omega)^{2}, \chi_{\mathrm{n} \lambda}=\varphi_{\mathrm{n} \lambda}+\gamma \sum_{j} \\
<\mathrm{j}\left|\mathrm{V}_{1}\right| \mathrm{n} \lambda>\varphi_{\mathrm{j}} /\left(\mathrm{E}_{\mathrm{n}}{ }^{(0)}-\mathrm{E}_{\mathrm{j}}{ }^{(0)}\right)+ \\
+\gamma \sum_{\mu \neq \lambda}\left\{\sum_{j}<\mathrm{n} \mu\left|\mathrm{V}_{1}\right| \mathrm{j}><\mathrm{j}\left|\mathrm{V}_{1}\right| \mathrm{n} \lambda>\varphi_{\mathrm{n} \mu} /\left[\left(\mathrm{E}_{\mathrm{n}}{ }^{(0)}-\mathrm{E}_{\mathrm{j}}{ }^{(0)}\right)\left(<\mathrm{n} \lambda\left|\mathrm{V}_{1}\right| \mathrm{n} \lambda>-<\mathrm{n} \mu\left|\mathrm{V}_{1}\right| \mathrm{n} \mu>\right)\right]\right. \\
\left.++<\mathrm{n} \mu\left|\mathrm{V}_{1}\right| \mathrm{n} \lambda>\varphi_{\mathrm{n} \mu} /\left(<\mathrm{n} \lambda\left|\mathrm{V}_{1}\right| \mathrm{n} \lambda>-<\mathrm{n} \mu\left|\mathrm{V}_{1}\right| \mathrm{n} \mu>\right)\right\}
\end{gathered}
$$

In Equation (8), $\lambda=(l, m), \mu=\left(l^{\prime \prime}, m^{\prime \prime}\right), j=\left(n^{\prime}, l^{\prime}, m^{\prime}\right), n^{\prime} \neq n$.

Substituting $V_{1}$ from Equation (5) in Equation (8), we obtain the following for $l>0$ :

$$
\mathrm{F}_{\mathrm{n} l \mathrm{~m}}=-\mathrm{Z}^{2} /\left(2 \mathrm{n}^{2}\right)+\mathrm{E}_{0}{ }^{2} /(2 \omega)^{2}+\left(\mathrm{Z}^{4} \mathrm{E}_{0}{ }^{2} / \omega^{4}\right)\left[3 \mathrm{~m}^{2}-l(l+1)\right] /\left[\mathrm{n}^{3}(2 l+3)(l+1)(2 l+1) l(2 l-1)\right]
$$

For finding $\mathrm{F}_{\mathrm{n} l \mathrm{~m}}$ (i.e., for $l=0$ ), instead of the formula for $\mathrm{V}_{1}$ from Equation (5), we use the following expression:

$$
\mathrm{V}_{1}=\mathrm{Z}\left[1-3 \cos ^{2} \theta+\varepsilon\left(4 \cos ^{2} \theta-1\right)\right] \mathrm{r}^{\varepsilon-3},|\varepsilon|<<1
$$

The expression for $\mathrm{V}_{1}$ from Equation (10) corresponds to the quasi-Coulomb nuclear potential $-\mathrm{Zr}^{\varepsilon-1}$. This trick allows removing the uncertainty that would otherwise arise while calculating matrix elements of the operator $V_{1}$ in the basis of the eigenfunctions $\varphi_{\mathrm{n} 00}$. For completeness we note that a similar uncertainty arises while calculating matrix elements $\left\langle\mathrm{n} l \mathrm{~m}\left|\mathrm{~V}_{2}\right| \mathrm{n}^{\prime} l^{\prime} \mathrm{m}\right\rangle$; in this case one should use the expression

$$
\left.\mathrm{V}_{2}=3 Z\left\{-3+30 \cos ^{2} \theta-35 \cos ^{4} \theta+\varepsilon\left[4-46 \cos ^{2} \theta+(176 / 3) \cos ^{4} \theta\right]\right]\right\} /\left(4 \mathrm{r}^{5-\varepsilon}\right)
$$

corresponding to the quasi-Coulomb nuclear potential $-\mathrm{Zr}^{\varepsilon-1}$.

After calculating the necessary matrix elements by using the potential $V_{1}$ from Equation (10) and then setting $\varepsilon=0$, we obtain the following result for the energy $\mathrm{F}_{\text {n00 }}$ (i.e., for $l=0$ ):

$$
F_{n 00}=-Z^{2} /\left(2 n^{2}\right)+E_{0}^{2} /(2 \omega)^{2}+Z^{4} E_{0}^{2} /\left(3 n^{3} \omega^{4}\right)
$$

It is worth noting that the above rigorously calculated result for $\mathrm{F}_{\mathrm{n} 00}$ can be also formally obtained from the right side of Equation (9) in the following three steps:

(1) set $\mathrm{m}=0$;

(2) cancel out $l(l+1)$ in the numerator and denominator;

(3) $\quad$ set $l=0$ 
Thus, the expression for quasienergies from paper [5] is not limited by $\mathrm{n}=1$ and $\mathrm{n}=2$ (as asserted in paper [5]). The above proves that they are actually applicable for any $n$.

For the validity of the above results, it is necessary that the characteristic value of the splitting of the energy level of the principal quantum number $n$, determined by Equations (9) and (12), significantly exceeded the energy shift $\Delta_{\mathrm{n} l m}$ caused by the term $\mathrm{H}_{1, \mathrm{osc}}$ in Equation (4). By limiting ourselves by the term in $\mathrm{H}_{1, \mathrm{osc}}$, containing the small parameter $\gamma$ in the lowest degree (i.e., by the term proportional to $\left.\gamma^{1 / 2} / \omega\right)$ in the high-frequency limit defined by Equation (1), we obtain the following relation (see [14]):

$$
\Delta_{\mathrm{n} l \mathrm{~m}}=-\mathrm{ZE}_{0}^{(2)} /\left(2 \omega^{6}\right) \sum_{n \neq n}|<\mathrm{n} l \mathrm{~m}| \mathrm{r}^{-2} \cos \theta|\mathrm{n} l \mathrm{~m}>|^{2}\left(\mathrm{E}_{\mathrm{n}}{ }^{(0)}-\mathrm{E}_{\mathrm{n}}{ }^{(0)}\right)
$$

that serves for finding the lower limit of validity with respect to the laser frequency $\omega$.

The above results were obtained for a linearly-polarized high-frequency laser field. A more general case where the high-frequency laser field is elliptically-polarized was considered in paper [2]. The vector-potential of the laser electric field was chosen in the form

$$
\mathbf{A}(\mathrm{t})=\mathrm{A}_{0}\left(1+\zeta^{2}\right)(\cos \omega \mathrm{t}, \zeta \sin \omega \mathrm{t}, 0), \mathrm{A}_{0}=-\mathrm{cE}_{0} / \omega
$$

where $\zeta$ is the ellipticity degree. For the quasi-Coulomb nuclear potential $-\mathrm{Zr}^{\varepsilon-1}$, the analog of the $\gamma \mathrm{V}_{1}$ from Equation (5), denoted below as $\mathrm{V}$, now can be represented as follows

$$
\mathrm{V}=\left[\mathrm{Z} /\left(2 \mathrm{c}^{2}\right)\right](\varepsilon+1)<\mathbf{B}^{2}>/ \mathrm{r}^{3+\varepsilon},|\varepsilon|<<1
$$

In Equation (15), vector $\mathbf{B}(\mathrm{t})$ is the solution of the equation

$$
\mathrm{d} \mathbf{B}(\mathrm{t}) / \mathrm{dt}=\mathbf{A}(\mathrm{t})
$$

and has the zero time average. The notation $<\ldots>$ stands for the average over the period of the laser field.

Substituting Equation (14) in Equation (15) and using the spherical coordinates, in paper [2] it was found the following:

$$
\mathrm{V}\left(\zeta^{2}\right)=-\left[\mathrm{ZE}_{0}^{2} /\left(8 \omega^{4} \mathrm{r}^{3+\varepsilon}\right)\right]\left\{\left(1-3 \cos ^{2} \theta\right)-2 \varepsilon \cos 2 \theta+\left[3\left(1-\zeta^{2}\right) /\left(1+\zeta^{2}\right)\right] \sin ^{2} \theta \cos 2 \varphi\right\}
$$

In paper [15], it was shown that

$$
\int_{0}^{\infty} \mathrm{r}^{-\mathrm{s}} \mathrm{R}_{\mathrm{n} l}(\mathrm{r}) \mathrm{R}_{\mathrm{n} l}(\mathrm{r}) \mathrm{r}^{2} \mathrm{dr}=0, \quad \mathrm{~s}=2,3,4, \ldots, l-l+1
$$

where $\mathrm{R}_{\mathrm{n} l}(\mathrm{r})$ are the radial wave functions of the hydrogenic atom/ion. Therefore, the matrix elements of the operator $\mathrm{V}\left(\zeta^{2}\right)$ satisfy the following relation:

$$
<\mathrm{n} l \mathrm{~m}\left|\mathrm{~V}\left(\zeta^{2}\right)\right| \mathrm{n} l^{\prime} \mathrm{m}^{\prime}>=0, \quad l^{\prime}=l \pm 2
$$

For a particular case of the circular polarization $\left(\zeta^{2}=1\right)$, the term containing $\cos 2 \varphi$ in Equation (17) is absent. Therefore, for the case of the circular polarization, due to the relation (19), the spherical eigenfunctions $\varphi_{\mathrm{n} / \mathrm{m}}$ are the correct eigenfunctions of the zero order for the perturbed Hamiltonian (as it was the case for the linear polarization of the laser field). The following energy eigenvalues correspond to these eigenfunctions:

$$
\mathrm{F}_{\mathrm{n} l \mathrm{~m}}=-\mathrm{Z}^{2} /\left(2 \mathrm{n}^{2}\right)+\mathrm{E}_{0}^{2} /(2 \omega)^{2}+<\mathrm{n} l \mathrm{~m}|\mathrm{~V}| \mathrm{n} l \mathrm{~m}>
$$




$$
\begin{gathered}
<\mathrm{n} l \mathrm{~m}|\mathrm{~V}| \mathrm{n} l \mathrm{~m}>=-\left[\mathrm{Z}^{4} \mathrm{E}_{0}{ }^{2} /\left(2 \omega^{4}\right)\right]\left[3 \mathrm{~m}^{2}-l(l+1)\right] /\left[\mathrm{n}^{3}(2 l+3)(l+1)(2 l+1) l(2 l-1)\right], l>0, \\
<\mathrm{n} l \mathrm{~m}|\mathrm{~V}| \mathrm{n} l \mathrm{~m}>=-\mathrm{Z}^{4} \mathrm{E}_{0}{ }^{2} /\left(6 \mathrm{n}^{3} \omega^{4}\right), l=0
\end{gathered}
$$

(In Equation (22), we corrected a misprint from the corresponding expression in Equation (10) from paper [2].) Just as in the case of the linear polarization of the laser field, the result presented in Equation (22) can be obtained from the right side of Equation (21) in the following three steps:

(1) set $\mathrm{m}=0$;

(2) cancel out $l(l+1)$ in the numerator and denominator;

(3) $\operatorname{set} l=0$

So, the expression for quasienergies from paper [5] for the circular polarization of the laser field is not limited by $n=1$ and $n=2$ (as asserted in paper [5]). The above proves that they are actually applicable for any $n$-just as it was in the case of the linear polarization of the laser field.

Now, we are coming back to the situation where the ellipticity degree of the laser field is arbitrary. When $\zeta^{2} \neq 1$, the term containing $\cos 2 \varphi$ in Equation (17) will couple the states $\varphi_{\mathrm{n} l \mathrm{~m}}$ and $\varphi_{\mathrm{n} l \mathrm{~m}^{\prime}}\left(l>0, \mathrm{~m}^{\prime}\right.$ $=\mathrm{m}-2$ ). However, the state $\varphi_{\mathrm{n} 00}$ would not be coupled by this term, and the state $\varphi_{\mathrm{n} 10}$ would not be coupled by this term either. Therefore, the energy eigenvalues for these two states do not depend on the ellipticity degree $\zeta$, and are still given by Equations (21) and (22).

For finding the eigenvalues of the energy for the other states in the general case of $\zeta^{2} \neq 1$, one should solve the corresponding secular equation. For the states $\varphi_{n l 1}$ and $\varphi_{n l-1}(l=1,2)$, the secular equation is a quadratic one, yielding the following two energy eigenvalues $F_{n s}(s=1,2)$ :

$$
\mathrm{F}_{\mathrm{ns}}=-\mathrm{Z}^{2} /\left(2 \mathrm{n}^{2}\right)+\mathrm{E}_{0}^{2} /(2 \omega)^{2}++\left\{\mathrm{Z}^{4} \mathrm{E}_{0}^{2} /\left[2 \omega^{4} \mathrm{n}^{3}(2 l+3)(2 l+1)(2 l-1)\right]\right\}\left[\left(l^{2}+l-3\right) /(l+1)+(-1)^{\mathrm{s}+1}(3 / 2)\left(1-\zeta^{2}\right) /\left(1+\zeta^{2}\right)\right]
$$

where $l=1,2 ; \mathrm{s}=1,2$. The corresponding eigenfunctions are

$$
\varphi_{\mathrm{ns}}=2^{-1 / 2}\left[(-1)^{\mathrm{s}+1} \varphi_{\mathrm{n} l 1}+\varphi_{\mathrm{n} l-1}\right], l=1,2 ; \mathrm{s}=1,2
$$

For the states $\varphi_{\mathrm{n} l 0}, \varphi_{\mathrm{n} l 2}$, and $\varphi_{\mathrm{n} l-2}(l=2,3)$, the secular equation is a cubic one, yielding the following three energy eigenvalues $F_{n p}(p=1,2,3)$

$$
\mathrm{F}_{\mathrm{n} 1}=-\mathrm{Z}^{2} /\left(2 \mathrm{n}^{2}\right)+\mathrm{E}_{0}{ }^{2} /(2 \omega)^{2}+\mathrm{V}_{22}, \mathrm{~F}_{\mathrm{np}}=-\mathrm{Z}^{2} /\left(2 \mathrm{n}^{2}\right)+\mathrm{E}_{0}^{2} /(2 \omega)^{2}+(1 / 2)\left(\mathrm{V}_{11}+\mathrm{V}_{22}+(-1)^{\mathrm{p}}\left[\left(\mathrm{V}_{11}-\mathrm{V}_{22}\right)^{2}+8 \mathrm{~V}_{12}{ }^{2}\right]^{1 / 2}, \mathrm{p}=2,3,\right.
$$

where

$$
\begin{gathered}
\mathrm{V}_{11}=\mathrm{Z}^{4} \mathrm{E}_{0}^{2} /\left[\left(2 \omega^{4} \mathrm{n}^{3}(2 l+3)(2 l+1)(2 l-1)\right]\right. \\
\mathrm{V}_{22}=-\mathrm{Z}^{4} \mathrm{E}_{0}^{2}[12-l(l+1)] /\left[\left(2 \omega^{4} \mathrm{n}^{3}(2 l+3)(2 l+1)(2 l-1) l(l+1)\right]\right. \\
\mathrm{V}_{12}=\left[3 \mathrm{Z}^{4} \mathrm{E}_{0}^{2} /\left(4 \omega^{4}\right)\right]\left[\left(1-\zeta^{2}\right) /\left(1+\zeta^{2}\right)\right]\{(l-1)(l+2) /[l(l+1)]\} /\left[\mathrm{n}^{3}(2 l+3)(2 l+1)(2 l-1)\right]
\end{gathered}
$$

The corresponding eigenfunctions are as follows

$$
\varphi_{\mathrm{n} 1}=2^{-1 / 2}\left[(-1)^{\mathrm{s}+1} \varphi_{\mathrm{n} l 2}-\varphi_{\mathrm{n} l-2}\right], \varphi_{\mathrm{np}}=\mathrm{a}(\mathrm{p}) \varphi_{\mathrm{n} l 0}+\mathrm{b}(\mathrm{p})\left(\varphi_{\mathrm{n} l 2}+\varphi_{\mathrm{n} l-2}\right), \mathrm{p}=2,3
$$

where

$$
\mathrm{a}(\mathrm{p})=2^{-1 / 2}\left\{1+(-1)^{\mathrm{p}+1}\left(\mathrm{~V}_{22}-\mathrm{V}_{11}\right) /\left[\left(\mathrm{V}_{11}-\mathrm{V}_{22}\right)^{2}+8 \mathrm{~V}_{12}{ }^{2}\right]^{1 / 2}\right\}^{1 / 2}, \mathrm{~b}(\mathrm{p})=\left[(-1)^{\mathrm{p} / 2]}\right]\left[1+(-1)^{\mathrm{p}}\left(\mathrm{V}_{22}-\mathrm{V}_{11}\right) /\left[\left(\mathrm{V}_{11}-\mathrm{V}_{22}\right)^{2}+8 \mathrm{~V}_{12}{ }^{2}\right]^{1 / 2}\right\}^{1 / 2}
$$

In summary, expressions (9), (12), (20)-(30) determine the splitting of hydrogenic spectral lines in the high-frequency laser field. The intensities of the split components can be calculated by using the well-known expressions (e.g., from book [16]) for the matrix elements 
$|<\mathrm{n} l \mathrm{~m}| \mathrm{r}^{-2} \cos \theta\left|\mathrm{n}^{\prime} l^{\prime} \mathrm{m}>\right|^{2}$ in the spherical coordinates. In particular, the allowance of the terms $\sim \gamma$ in Equation (8) would lead to the appearance of the forbidden components (of intensities $\sim \gamma^{2}$ ) in the spectra of hydrogenic lines. In general, the observation of the splitting of the spectrum of a hydrogenic line in two different polarizations, allows determining the following three physical quantitites:

(1) the polarization of the vector-potential $\mathbf{A}(\mathrm{t})$;

(2) the ellipticity degree $\zeta$;

(3) the amplitude $E_{0}$ of the laser field (for the known laser frequency $\omega$ ).

This is possible because, for the known laser frequency $\omega$, the splitting of a hydrogenic line depends on two parameters: the amplitude $\mathrm{E}_{0}$ of the laser field and the ellipticity degree $\zeta$. Therefore, the experimental splitting in two different polarizations would provide two equations for determining the two unknown quantities- $\mathrm{E}_{0}$ and $\zeta$.

\section{Classical (Rydberg) Hydrogen Atoms in a High-Frequency Laser Field: Celestial Analogies}

We consider the situation where the laser frequency $\omega$ is much greater than the Kepler frequency $\omega_{\mathrm{K}}=\mathrm{m}_{\mathrm{e}} \mathrm{e}^{4} /\left(\mathrm{n}^{3} \hbar^{3}\right)$ of the highly excited (Rydberg) hydrogen atom.

$$
\omega>>\omega_{K}
$$

Here, $\mathrm{m}_{\mathrm{e}}$ and e are the electron mass and charge, respectively; $\mathrm{n}>>1$ is the principal quantum number. Under the condition (31), the laser field constitutes the fast subsystem, while the Rydberg atom constitutes the slow subsystem.

The Kapitza's method of splitting the motion into "fast" and "slow" variables and introducing the concept of an effective potential for the slow subsystem, in its standard form [17-19], is not applicable for the case of a spatially uniform amplitude $f$ of the oscillatory force $f\left(x_{\alpha}\right) \cos \omega t$, as mentioned in Chapter 1. A generalization of the Kapitza's effective potential for a spatially uniform force amplitude $\mathbf{f}$, has been provided by Nadezhdin and Oks [3] and is presented below.

We consider a particle in a static potential $U\left(x_{\alpha}\right)$ and under the force $f\left(x_{\alpha}\right) \cos \omega t$. We seek the solution of the equation of the motion

$$
m d^{2}\left(x_{\alpha}\right) / d t^{2}=-d U / d x_{\alpha}+f_{\alpha} \cos \omega t
$$

in the form

$$
\mathrm{x}_{\alpha}(\mathrm{t})=\mathrm{X}_{\alpha}(\mathrm{t})+\xi_{\alpha}(\mathrm{t})
$$

and expand the right side in powers of the rapid oscillations $\xi_{\alpha}$

$$
\mathrm{md}^{2}\left(\mathrm{X}_{\alpha}\right) / \mathrm{dt}^{2}+\mathrm{md}^{2}\left(\xi_{\alpha}\right) / \mathrm{dt}^{2}=-\mathrm{dU} / \mathrm{dX} \mathrm{X}_{\alpha}-\xi_{\alpha} \mathrm{d}^{2} \mathrm{U} / \mathrm{dX}_{\alpha} \mathrm{dX} \mathrm{X}_{\beta}-(1 / 2) \xi_{\beta} \xi_{\gamma} \mathrm{d}^{3} \mathrm{U} / \mathrm{dX} \mathrm{X}_{\alpha} \mathrm{dX}_{\beta} \mathrm{dX} \mathrm{X}_{\gamma}+\left[\mathrm{f}_{\alpha}(\mathbf{X})+\xi_{\beta} \mathrm{df_{ \alpha } / \mathrm { dX }} \mathrm{X}_{\beta}\right] \cos \omega \mathrm{t}
$$

Here and below, the summation over repeated subscripts is understood.

For the oscillatory terms, it is sufficient to write

$$
m d^{2}\left(\xi_{\alpha}\right) / d t^{2}=f_{\alpha}(X) \cos \omega t
$$

so that

$$
\xi_{\alpha}=-\left[\mathrm{f}_{\alpha} /\left(\mathrm{m} \omega^{2}\right)\right] \cos \omega \mathrm{t}
$$

Substituting Equation (36) in Equation (34) and averaging over the period $2 \pi / \omega$, we obtain the equation for the averaged motion $\mathbf{X}(\mathrm{t})$

$$
m d^{2}\left(X_{\alpha}\right) / d t^{2}=-d U / d X_{\alpha}-\left[1 /\left(4 m^{2} \omega^{4}\right)\right] f_{\beta} f_{\gamma} d^{3} U / d X_{\alpha} d X_{\beta} d X_{\gamma}-\left[1 /\left(2 m \omega^{2}\right)\right] f_{\beta} d f_{\alpha} / d X_{\beta}
$$


For a spatially uniform force $\mathbf{f}$, the term proportional to $1 / \omega^{2}$ in Equation (37) vanishes, so that it becomes important to take into account the term proportional to $1 / \omega^{4}$. As a result, the equation for the averaged motion takes the form

$$
m d^{2}\left(X_{\alpha}\right) / d t^{2}=-d / d X_{\alpha}\left\{U+\left[f_{\beta} f_{\gamma} /\left(4 m^{2} \omega^{4}\right)\right] d^{2} U / d X_{\beta} d X_{\gamma}\right\}
$$

Thus, in this situation we deal with the effective potential

$$
\mathrm{U}_{\text {eff }}=\mathrm{U}+\left[\mathrm{f}_{\beta} \mathrm{f}_{\gamma} /\left(4 \mathrm{~m}^{2} \omega^{4}\right)\right] \mathrm{d}^{2} \mathrm{U} / \mathrm{dX} \mathrm{X}_{\beta} \mathrm{dX}
$$

Oks, Davis, and Uzer [4] applied the effective potential from Equation (39) to the case of a Rydberg hydrogen atom in an elliptically-polarized high-frequency laser field:

$$
\mathbf{E}(t)=\mathbf{e}_{\mathbf{x}} \varepsilon_{0} \cos \omega t+\mathbf{e}_{\mathbf{y}} \mu \varepsilon_{0} \sin \omega t
$$

where $\mu$ is the ellipticity degree. The peak field $\varepsilon_{0}$ in expression (40) is connected with the time-average of the electric field as follows:

$$
<\mathrm{E}^{2}(\mathrm{t})>=<\left\{\varepsilon_{0}^{2} \cos ^{2} \omega \mathrm{t}+\mu^{2} \varepsilon_{0}^{2} \sin ^{2} \omega \mathrm{t}\right\}>=\varepsilon_{0}^{2}\left(1+\mu^{2}\right) / 2
$$

On the other hand, one can define an effective amplitude $\mathrm{E}_{0}$ through

$$
<\mathrm{E}^{2}(\mathrm{t})>=\mathrm{E}_{0}^{2} / 2
$$

By equating the right sides of Equations (41) and (42), one obtains the following relation between $\mathrm{E}_{0}$ and $\varepsilon_{0}$

$$
\varepsilon_{0}=\mathrm{E}_{0} /\left(1+\mu^{2}\right)^{\frac{1}{2}}
$$

By applying the effective potential from Equation (39) to the case of the high-frequency elliptically polarized laser field from Equation (40), Oks, Davis, and Uzer [4] found the following effective potential:

$$
\mathrm{U}_{\mathrm{eff}}=-\mathrm{e}^{2} / \mathrm{r}+\left(\gamma / \mathrm{r}^{3}\right)\left\{\left(1+\mu^{2}\right)-3 \sin ^{2} \theta\left[1-\left(1-\mu^{2}\right) \sin ^{2} \varphi\right]\right\}
$$

where

$$
\gamma=\left(\mathrm{e}^{4} \varepsilon_{0}{ }^{2}\right) /\left(4 \mathrm{~m}^{2} \omega^{4}\right)
$$

$\theta$ is the polar angle, and $\varphi$ is the azimuthal angle of the radius vector of the electron (the z-axis is chosen to be perpendicular to the polarization plane).

In the particular case where the high-frequency laser field is circularly polarized $(\mu=1)$, the effective potential from Equation (44) simplifies to

$$
\mathrm{U}_{\text {eff }}(\mathrm{r}, \theta)=-\mathrm{e}^{2} / \mathrm{r}+\left(\gamma / \mathrm{r}^{3}\right)\left(3 \cos ^{2} \theta-1\right)
$$

For this case of the circular polarization, by using Equation (43) with $\mu=1$, formula (45) for $\gamma$ can be also expressed through the time-average square of the laser field as follows:

$$
\gamma=\left(\mathrm{e}^{4} \mathrm{E}_{0}^{2}\right) /\left(8 \mathrm{~m}^{2} \omega^{4}\right)
$$

The effective potential given in Equation (46) has the following remarkable feature: it is identical to the potential of a satellite orbiting a prolate planet. The motion of this satellite has been completely investigated in celestial mechanics (see, e.g., book [20], Section 10.4). It turns out that not only does the ellipse precesses in its plane with some frequency $f_{e}<<\Omega$, but the plane of orbit precesses as well with frequency $\mathrm{f}_{\mathrm{p}} \sim \mathrm{f}_{\mathrm{e}}$. 
The precessions above are found via canonical perturbation theory and by employing action-angle formulation (see, e.g., book [21]). In this situation the effective potential from Equation (31) can be represented in the form of the following perturbation Hamiltonian

$$
\Delta \mathrm{H}=\mathrm{C}\left(3 \cos ^{2} \theta-1\right) / \mathrm{r}^{3}, \mathrm{C}=\mathrm{k}^{2}\left(\mathrm{I}_{3}-\mathrm{I}_{1}\right) /\left(2 \mathrm{M}_{0}\right), \mathrm{k}=\mathrm{GM}_{0} \mathrm{~m}
$$

where $\mathrm{m}$ is the mass of the satellite, $\mathrm{M}_{0}$ is the mass of the a prolate planet mass, and $\mathrm{I}_{3}$ and $\mathrm{I}_{1}$ are the principal moments of inertia, $\mathrm{I}_{1}$ being the moment of inertia with respect to the axis of symmetry. The time average of the perturbation Hamiltonian is

$$
<\Delta \mathrm{H}>=\left[\mathrm{m}^{2} \mathrm{k}^{2}\left(\mathrm{I}_{3}-\mathrm{I}_{1}\right) /\left(2 \mathrm{M}_{0} \mathrm{M}^{3} \tau\right)\right] \int_{0}^{2 \pi}(1+\varepsilon \cos \Psi)\left(3 \cos ^{2} \theta-1\right) \mathrm{d} \Psi
$$

where $\varepsilon$ is the eccentricity of the satellite orbit, $M$ is the magnitude of the total angular momentum, $\tau$ is the period, and $\Psi$ is the angle of the radius-vector in the orbital plane relative to the periapsis. We use the relation

$$
\left.3 \cos ^{2} \theta-1=\left[(1 / 2)-(3 / 2) \cos ^{2} i\right)\right]-\left\{(3 / 2) \sin ^{2} i \cos [2(\Psi+\omega)]\right\}
$$

where $i$ is the angle between the unperturbed plane of orbit and the equatorial plane of the planet (the angle of inclination). Employing Equation (50), we find

$$
<\Delta \mathrm{H}>=\pi \mathrm{m}^{2} \mathrm{k}^{2}\left(\mathrm{I}_{3}-\mathrm{I}_{1}\right)\left(1-3 \cos ^{2} \mathrm{i}\right) /\left(2 \mathrm{M}_{0} \mathrm{M}^{3} \tau\right) . .
$$

We relate the variable $i$ to the action angle variables $\mathrm{J}_{1}$ and $\mathrm{J}_{2}$ as

$$
\mathrm{J}_{1} / \mathrm{J}_{2}=\operatorname{cosi}, \mathrm{J}_{1}=\mathrm{J}_{\varphi}=2 \pi \mathrm{M}_{\mathrm{z}}
$$

where $\mathrm{M}_{\mathrm{z}}$ is the constant value of angular momentum about the polar axis,

$$
\mathrm{J}_{2}=\mathrm{J}_{\varphi}+\mathrm{J}_{\theta}=2 \pi \mathrm{M}
$$

Here, $\mathrm{M}$ is the magnitude of the total angular momentum, and

$$
\mathrm{J}_{3}=\pi \mathrm{k}\left[(2 \mathrm{~m} /(-\mathrm{E})]^{\frac{1}{2}}\right.
$$

where $\mathrm{k}$ is the constant from the expression for the unperturbed gravitational potential

$$
\mathrm{V}(\mathrm{r})=-\mathrm{k} / \mathrm{r}
$$

and corresponds to $\mathrm{e}^{2}$ in the atomic problem.

The orbit undergoes two precessions, as follows. Because of the smallness of the perturbation, the precession of the orbital plane around the polar axis shows up as a secular change in $\Omega$.

$$
<\mathrm{d} \Omega / \mathrm{dt}>\tau /(2 \pi)=\left[\partial(\Delta \mathrm{H}) / \partial \mathrm{J}_{1}\right] \tau /(2 \pi)=[\tau /(2 \pi \mathrm{M})] \partial(\Delta \mathrm{H}) / \partial(\operatorname{cosi})
$$

Therefore, it follows that the frequency of precession of the orbital plane around the polar axis is given by

$$
f_{p}=-(3 / 2)\left(I_{3}-I_{1}\right) \operatorname{cosi} /\left[M_{0} a^{2}\left(1-\varepsilon^{2}\right)^{2}\right]
$$

where we have made use of the expression

$$
\mathrm{M}^{2}=\operatorname{mka}\left(1-\varepsilon^{2}\right)
$$


(Here, a is the semi-major axis.)

The second one is the precession of the periapsis of the elliptical orbit in the plane of orbit. It is given by

$$
<\mathrm{d} \omega / \mathrm{dt}>\tau /(2 \pi) \equiv\left[\partial(\Delta \mathrm{H}) / \partial \mathrm{J}_{2}\right] \tau /(2 \pi)=[\tau /(2 \pi)] \partial(\Delta \mathrm{H}) / \partial \mathrm{M}
$$

Upon taking the derivatives, we find

$$
f_{e}=3\left(I_{3}-I_{1}\right)\left(5 \cos ^{2} i-1\right) /\left[4 M_{0} a^{2}\left(1-\varepsilon^{2}\right)^{2}\right]
$$

The averaged Hamiltonian $<\Delta \mathrm{H}>$ from Equation (51) does not depend on the angular variables of the spherical coordinate system. Consequently, the action variables $\mathrm{J}_{1}, \mathrm{~J}_{2}, \mathrm{~J}_{3}$ are conserved with respect to the averaged motion. The major semi-axis a and the eccentricity $\varepsilon_{0}$ of the orbit are the following functions of $\mathrm{J}_{1}, \mathrm{~J}_{2}, \mathrm{~J}_{3}$

$$
\mathrm{a}=\mathrm{J}_{3}{ }^{2} /\left(4 \pi^{2} \mathrm{mk}\right), \varepsilon=\left[\left(1-\left(\mathrm{J}_{2} / \mathrm{J}_{3}\right)^{2}\right)\right]^{\frac{1}{2}}
$$

Therefore, there is no secular change in either a or $\varepsilon$. Physically this means that the shape and size of this precessing ellipse, when averaged over the orbital period, will not change in time.

The approximate conservation of the shape and size of the precessing ellipse means that the square of the angular momentum $\mathbf{M}^{2}$ is an approximately conserved quantity (to within the accuracy of terms $\sim \mathrm{E}_{0}^{2}$ ). Physically this means that the averaged system has a higher symmetry than the geometrical symmetry, which was axial symmetry. In other words, the system has an algebraic symmetry which is spherical.

Thus, not only does the unperturbed Hamiltonian $\mathrm{H}_{0}$ commute with $\mathrm{M}^{2}$ and $\mathrm{M}_{\mathrm{z}}$, but the perturbed Hamiltonian $\mathrm{H}_{0}+\mathrm{V}$ commutes (approximately) with $\mathrm{M}^{2}$ and $\mathrm{M}_{\mathrm{z}}$. This justifies the employment of the non-degenerate classical perturbation theory in the previous section.

This also means that, in the quantum treatment of the corresponding atomic problem, the perturbation $\mathrm{V}$ is diagonal (in the same approximation) in the basis of the spherical wave functions. Consequently, due to the conservation of $\mathbf{M}^{2}$, the perturbed energies can be found as mean values of the perturbation over the unperturbed motion. Following paper [4], we find the quantum corrections to the energy levels in the quasiclassical formalism as follows.

The perturbation term in the effective potential is

$$
\Delta \mathrm{U}=\left[\gamma / \mathrm{r}^{3}\right]\left[3 \cos ^{2} \theta-1\right]
$$

We average this expression over the period of the unperturbed motion

$$
<\Delta \mathrm{U}>=[\gamma / \tau] \int_{0}^{2 \pi}\left[3 \cos ^{2} \theta-1\right] \mathrm{r}^{-3} \mathrm{dt}
$$

The angular momentum can be written as $\mathrm{M}=m r^{2} \mathrm{~d} \Psi / \mathrm{dt}$, where $\Psi$ is the angle of the radius vector in the orbital plane relative to the periapsis. Substituting the expression

$$
\mathrm{dt}=m r^{2} \mathrm{M}^{-1} \mathrm{~d} \Psi
$$

into Equation (63) for the effective potential, we arrive at the following

$$
<\Delta \mathrm{U}>=[\gamma \mathrm{m} /(\mathrm{M} \tau)] \int_{0}^{2 \pi}\left(3 \cos ^{2} \theta-1\right) \mathrm{r}^{-1} \mathrm{~d} \Psi
$$

Using the relations

$$
\cos \theta=\operatorname{sini} \cos \Psi, \mathrm{r}^{-1}=(1+\varepsilon \cos \Psi) \mathrm{p}^{-1}, \mathrm{p}=\mathrm{M}^{2}(\mathrm{mk})^{-1}
$$


where $\mathrm{k}=\mathrm{e}^{2}$, we find

$$
<\Delta \mathrm{U}>=\left[\gamma \mathrm{m}^{2} /\left(\mathrm{M}^{3} \tau\right)\right] \int_{0}^{2 \pi}\left(3 \sin ^{2} \mathrm{i} \cos ^{2} \Psi-1\right)(1+\varepsilon \cos \Psi) \mathrm{d} \Psi=\left[\left(\gamma \mathrm{m}^{2} \mathrm{e}^{2}\right) /\left(\mathrm{M}^{3} \tau\right)\right] 2 \pi\left[(3 / 2) \sin ^{2} \mathrm{i}-1\right]
$$

Substituting the expressions for the period

$$
\tau=2 \pi \mathrm{n}^{3} \hbar^{3} /\left(\mathrm{me}^{4}\right)
$$

into Equation (67), we obtain

$$
<\Delta U>=\left[\gamma \mathrm{m}^{3} \mathrm{e}^{6}\right]\left[(3 / 2) \sin ^{2} \mathrm{i}-1\right] /\left[\mathrm{M}^{3} \hbar^{3} \mathrm{n}^{3}\right]
$$

Transforming

$$
\left[(3 / 2) \sin ^{2} \mathrm{i}-1\right]=(1 / 2)\left\{\left[3\left(1-\cos ^{2} \mathrm{i}\right)-2\right]\right\}, \cos \mathrm{i}=\mathrm{M}_{\mathrm{z}} / \mathrm{M}
$$

and replacing

$$
\mathrm{M}=\hbar\left(1+\frac{1}{2}\right), \mathrm{M}_{\mathrm{z}}=\mathrm{m}_{0} \hbar
$$

in Equation (67), we finally obtain the corrections to the energy levels

$$
\mathrm{E}_{\mathrm{n} l \mathrm{~m}}^{1} \equiv<\Delta \mathrm{U}>=\left[\left(\mathrm{e}^{10} \mathrm{mE}_{0}^{2}\right)\left(8 \hbar^{6} \mathrm{n}^{3} \omega^{4}\right)^{-1}\right]\left\{\left[\left(l+\frac{1}{2}\right)^{2}-3 \mathrm{~m}_{0}^{2}\right]\left[\left(l+\frac{1}{2}\right)^{-5}\right]\right\}
$$

We emphasize again that Equation (73) is valid for $n, l>>1$. Hence, the energy levels are split, and the degeneracy is partially removed. Because " $\mathrm{m}_{0}$," the magnetic quantum number, is squared, terms where the magnitude of $\mathrm{m}_{0}$ is the same, but the sign differs, will remain degenerate.

Paper [4] also studied the case where the ellipticity degree $\mu$ is arbitrary, but the Keplerian plane and the plane of electromagnetic radiation are coincident. This means that $\mathrm{M}_{\mathrm{z}}=\mathrm{M}$ and that the polar angle of the radius-vector does not vary in the course of time. In this case, the perturbed motion is really only two-dimensional because all of the forces acting on the particle are confined to the plane. The effective potential becomes

$$
\mathrm{U}_{\text {eff }}=-\mathrm{e}^{2} / \mathrm{r}+\left(\gamma / \mathrm{r}^{3}\right)\left[\left(1-2 \mu^{2}\right)-3\left(1-\mu^{2}\right) \cos \varphi\right]
$$

This problem is equivalent to a problem of celestial mechanics in which a satellite revolves in an equatorial orbit about a slightly non-spherical planet. For this case the plane of orbit does not change its orientation in the course of time. Therefore, the only precession that might occur is the precession of the periapsis of the ellipse. Further details can be found in paper [4].

Now we proceed to the case of a hydrogen Rydberg atom in a linearly-polarized high-frequency laser field $\mathbf{E}(\mathbf{t})=\mathbf{E}_{0} \cos \omega t$. For this case, by using the expression (39) for the effective potential as the starting point, Nadezhdin and Oks [3] obtained

$$
\mathrm{U}_{\text {eff }}(\mathrm{r}, \theta)=-\mathrm{e}^{2} / \mathrm{r}-\left(\gamma_{0} / \mathrm{r}^{3}\right)\left(3 \cos ^{2} \theta-1\right), \quad \gamma_{0}=\mathrm{e}^{4} \mathrm{E}_{0}^{2} /\left(4 \mathrm{~m}_{\mathrm{e}}{ }^{2} \omega^{4}\right)
$$

where $\theta$ is the polar angle, that is, the angle between the radius-vector $\mathbf{r}$ of the electron and the $\mathrm{z}$-axis chosen along the vector-amplitude $\mathbf{E}_{0}$ of the laser field.

It is remarkable that the effective potential from Equation (74) is mathematically equivalent to the effective potential of a satellite moving around an oblate planet, such as, e.g., the Earth. Indeed, because of this slightly flattened shape, the potential energy $U(r)$ of a satellite in the gravitational field of the Earth slightly differs from the potential energy $\mathrm{U}_{0}(\mathrm{r})=\mathrm{GmM} / \mathrm{r}$ it would have if the Earth would be a sphere. The perturbed potential energy $\mathrm{U}(\mathrm{r})$ can be approximately represented in the form

$$
\mathrm{U}(\mathrm{r}, \phi)=-(\mathrm{GmM} / \mathrm{r})\left[1-\mathrm{I}_{2}(\mathrm{R} / \mathrm{r})^{2} \mathrm{P}_{2}(\sin \phi)\right]
$$


(Equation (75) is approximate because it represents only the first two terms of the expansion of the potential energy in inverse powers of $\mathrm{r}$.) Here, $\mathrm{G}=6.6726 \times 10^{-8} \mathrm{~cm}^{3} /\left(\mathrm{s}^{2} \mathrm{~g}\right)$ is the gravitational constant (one of the fundamental constants of the nature); $\mathrm{m}$ and $\mathrm{M}$ are the masses of the satellite and the Earth, respectively; $\mathrm{I}_{2}=-1.082 \times 10^{-3}$ is a constant related to the slight difference between the equatorial and polar diameters of the Earth; $\mathrm{R}=6.378 \times 10^{8} \mathrm{~cm}$ is the equatorial radius of the Earth; $\theta$ is the geographical latitude of the satellite at any point of its orbit; and $\mathrm{P}_{2}(\sin \phi)$ is one of the Legendre polynomials.

$$
\left.P_{2}(\sin \phi)\right]=\left(3 \sin ^{2} \phi-1\right) / 2
$$

For the potential energy from Equation (75), there exists an exact analytical solution for the satellite motion. Details can be found, for example, in Section 1.7 of Beletsky's book [22]. The following outcome is similar, but not identical to the case discussed previously in this review, where a satellite moves around a prolate planet.

The elliptical orbit of the satellite undergoes two types of the precession simultaneously, but without changing its shape. The first one is the precession of the orbit in its plane. It occurs with the angular frequency

$$
\Omega_{\text {precession in plane }}=\left(3 I_{2} / 4\right)(\mathrm{R} / \mathrm{p})^{2}\left(1-5 \cos ^{2} \mathrm{i}\right) \omega_{\mathrm{K}}
$$

where $\mathrm{i}$ is the inclination, that is, the angle between the plane of the satellite orbit and the equatorial plane of the Earth. The quantity $p$ in Equation (77), it is related to parameters of the unperturbed elliptical orbit of the satellite as follows

$$
\mathrm{p}=2 \mathrm{r}_{\min } \mathrm{r}_{\max } /\left(\mathrm{r}_{\min }+\mathrm{r}_{\max }\right)
$$

where $r_{\min }$ and $r_{\max }$ are, respectively, the minimum and maximum distances of the satellite from the center of the Earth.

Interestingly enough, for $\mathrm{i}=63.4$ degrees, one gets $1-5 \cos ^{2} \mathrm{i}=0$, so that $\Omega_{\text {precession in plane }}$ vanishes. This means that satellites lunched at this inclination do not undergo the precession in the plane of the orbit.

The second simultaneous precession is the precession of the plane of the satellite orbit. The precession frequency is

$$
\Omega_{\text {precession of plane }}=\left(3 \mathrm{I}_{2} / 2\right)(\mathrm{R} / \mathrm{p})^{2}(\cos \mathrm{i}) \omega_{\mathrm{K}}
$$

At $\mathrm{i}=90$ degrees, one gets $\Omega_{\text {precession of plane }}=0$. For such inclination, which corresponds to the plane of orbit perpendicular to the Earth equator, there is no precession of the plane of orbit. These are so-called polar satellites.

Thus, the fact that the Earth is slightly flattened, does not affect the elliptical shape of the satellite orbit or the inclination of the orbit. It does result, generally, in two simultaneous precessions of the orbit with frequencies given by Equations (77) and (79).

Taking into account that the geographical latitude $\phi$ in Equation (75) and the polar angle $\theta$ in Equation (74) are related as $\phi=\pi / 2-\theta$ (where $\pi / 2$ is the 90 degrees angle expressed in radians), so that $\sin ^{2} \phi=\cos ^{2} \theta$, and that the quantity $I_{2}$ in Equation (75) is negative (so that $I_{2}=-\left|I_{2}\right|$ ), Equation (75) can be re-written in the form

$$
\mathrm{U}(\mathrm{r}, \theta)=-(\mathrm{GmM} / \mathrm{r})-\left(\mathrm{GmM}\left|\mathrm{I}_{2}\right| \mathrm{R}^{2} / \mathrm{r}^{3}\right)\left(3 \cos ^{2} \theta-1\right) / 2
$$

The comparison of Equation (74) and (80) shows that the potential energy of the electron in a hydrogen Rydberg atom under a high-frequency linearly polarized laser field is in fact mathematically equivalent to the potential energy of a satellite around the oblate Earth. Indeed, if in Equation (80) one would substitute GmM by e $\mathrm{e}^{2}$ and $\left|\mathrm{I}_{2}\right| \mathrm{R}^{2}$ by $2 \gamma / \mathrm{e}^{2}$, one would obtain Equation (74). 
Therefore, the motion of the electron in a hydrogen Rydberg atom under a high-frequency linearly polarized laser field can be described in the same way as the motion of a satellite around the oblate Earth. Namely, first of all, the shape of the elliptical orbit and the angle between the orbital plane and the plane perpendicular to the laser field amplitude $\mathbf{E}_{0}$ would not be affected by the laser field: they would remain the same in the course of time. The elliptical orbit of the electron undergoes two precessions simultaneously: the elliptical orbit precesses in its own plane with the frequency

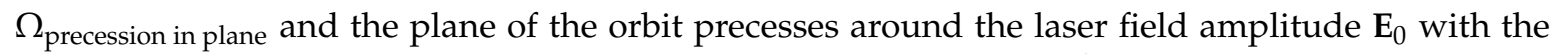

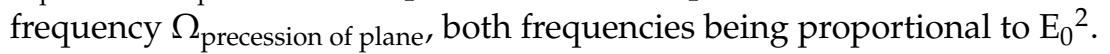

There is an interesting fact about the conserved quantities for both of these physical systems (just like in the case of the circular polarization of the laser field, discussed previously in this chapter). If the motion of a particle is characterized by a potential energy that depends only on the magnitude $r$ of the radius-vector $\mathbf{r}$ of the particle, but not on the direction of vector $\mathbf{r}$, then all directions in space are equivalent for such a system: the system is said to have the spherical symmetry. As the consequence of the spherical symmetry, the angular momentum vector $\mathbf{M}$ is conserved both by the magnitude and by the direction for such a system. If a linearly polarized laser field applied to such an atomic system, then there is no more the equivalence of all directions in space: the potential energy now depends not only on the magnitude $r$ of the radius-vector $\mathbf{r}$ but also on the angle $\theta$ between the vector $\mathbf{r}$ and the laser field amplitude vector $\mathbf{E}_{0}$. The latter vector defines the preferred direction in space. Only all directions in the plane perpendicular to the vector $\mathbf{E}_{0}$ remain equivalent: the symmetry of the system is said to be reduced from spherical to axial. As a consequence, only the angular momentum projection $\mathrm{M}_{\mathrm{z}}$ on the vector $\mathbf{E}_{0}$ remains conserved.

Similarly, because the Earth is not a sphere but an oblate spheroid, the axis connecting the two poles defines the preferred direction in space. Only the angular momentum projection $\mathrm{M}_{\mathrm{z}}$ on this direction remains conserved.

However, for the specific form of the potential energy dependence on $r$ and $\theta$, as in Equations (75) and (80), in addition to the exact conservation the angular momentum projection $\mathrm{M}_{\mathrm{z}}$, there is also the conservation of the square of the angular momentum $\mathrm{M}^{2}$. The latter quantity is proportional to the area of the orbit. Therefore, the fact that the shape of the elliptical orbit is not affected by the perturbation (by the laser field for the atomic system of by the spheroidness of the Earth in the case of the satellite) signifies the conservation of $\mathrm{M}^{2}$.

For completeness we note another physical system equivalent to the "satellite-spheroidal planet" system. Namely, nuclei of heavy atoms can have either the shape of a prolate spheroid or the shape of the oblate spheroid. So, the motion of the electron in a hydrogen Rydberg atom under a high-frequency laser field of the linear or circular polarizations also has the analogy with the motion of the electron in heavy hydrogenic ions.

It should be emphasized that there are hydrogenic atoms and ions where the negatively charged particle orbiting the nucleus is not the electron, but the muon. Muonic atoms and ions are more sensitive to the nuclear shape than the electronic atoms and ions. In particular, in paper [23] it was shown how the shift of spectral lines of muonic hydrogenic ions can serve for the experimental determination of the parameters of the nuclear shape (such as, for example, the parameter analogous to the constant $\mathrm{I}_{2}$ in Equation (75)).

The bottom line is that all the three above physical systems exhibit a higher than geometrical symmetry. This is an important result in its own right.

It should be emphasized that, for the type of the potential energies given by Equations (74) or (80), the square of the angular momentum $\mathrm{M}^{2}$ is conserved exactly. However, Equations (74) and (80) were obtained by neglecting some corrections (much smaller than the second term in (74) or in (80). Since Equations (74) and (80) are approximate, so is the conservation of $\mathrm{M}^{2}$. Nevertheless, any additional quantity that is conserved (whether exactly or approximately) is physically important. 


\section{Discussion and Conclusions}

Let us discuss now the advantages of this analytical method. We considered a hydrogen atom in a high frequency field

$$
\omega \gg \omega_{\mathrm{kn}}
$$

where $\omega_{\mathrm{kn}}$ is an atomic transition frequency. In the standard time-dependent perturbation theory, the transition probability can be written as (see, e.g., textbook [24])

$$
\left(\mathrm{dE}_{0}\right)^{2} /\left(\hbar^{2} \omega^{2}\right) \equiv \omega_{\mathrm{E}}^{2} / \omega^{2}
$$

where $\omega_{\mathrm{E}} \equiv\left(\mathrm{dE}_{0}\right) / \hbar$ may be interpreted as the peak frequency of precession of the dipole moment of the system. Since the atomic dipole moment can be estimated as $\mathrm{d} \sim \mathrm{n}^{2} \hbar^{2} /\left[\mathrm{me}^{2}\right]$, then

$$
\omega_{\mathrm{E}} \sim \mathrm{n}^{2} \hbar \mathrm{E}_{0} /(\mathrm{me})
$$

Thus, we arrive at the small parameter employed by standard perturbation theory

$$
\varepsilon_{\mathrm{pt}}=\omega_{\mathrm{E}}^{2} / \omega^{2} \ll 1
$$

In the advanced method of separating rapid and slow subsystems, the small parameter can be found from the ratio of the energy correction to the unperturbed energy.

$$
\varepsilon_{\text {our }}=\Delta \mathrm{E} / \mathrm{E}^{(0)}, \mathrm{E}^{(0)}=\mathrm{me}^{4} /\left(2 \hbar^{2} \mathrm{n}^{2}\right)
$$

Using the above results for $\Delta \mathrm{E}$, we find

$$
\Delta \mathrm{E} / \mathrm{E}^{(0)} \sim \mathrm{e}^{6} \mathrm{E}_{0}^{2} /\left(\hbar^{4} \mathrm{n}^{4} \omega^{4}\right)=\varepsilon_{\text {our }}
$$

or in atomic units

$$
\varepsilon_{\text {our }}=\mathrm{E}_{0}{ }^{2} /\left(\mathrm{n}^{4} \omega^{4}\right) \sim\left[\omega_{\mathrm{E}} /\left(\mathrm{n}^{4} \omega^{2}\right)\right]^{2} \sim \varepsilon_{\mathrm{pt}}{ }^{2}[\Omega /(\mathrm{n} \omega)]^{2}
$$

where $\Omega=\left(E_{n+1}{ }^{(0)}-E_{n}{ }^{(0)}\right) / \hbar=n^{-3}$ is the Kepler frequency. Thus, for the high frequency case $(\Omega \ll \omega)$, even when the method of the standard perturbation theory is no longer applicable, i.e., when $\varepsilon_{\mathrm{pt}} \sim 1$, the advanced method of separating rapid and slow subsystems remains valid.

Finally, let us discuss the connection between the above results and the transition to chaos. Multidimensional problems, like those presented above, play the crucial role in the fascinating transition regime between classical and quantum mechanics. In this context, paper [4] addressed the dynamics of Rydberg electrons placed in high-frequency circularly polarized and elliptically polarized "laser" (microwave) fields. These problems were mapped onto problems well-known from celestial mechanics, discovering approximate constants of the motion in the process. It was shown how the dynamics of a Rydberg electron, whose orbital plane differs from the plane of polarization of circularly polarized radiation, can be mapped on the problem of a satellite orbiting a prolate planet. Although the angular momentum precesses, its magnitude is an approximate constant. Similarly, when the electron's orbit plane coincides with the plane of elliptically polarized microwaves, it moves like a satellite orbiting a slightly non-spherical planet in its equatorial plane. In this case, the z-component of the angular momentum emerges as a hidden symmetry.

The high-frequency case has received less attention than the low-frequency, resonant cases, and therefore it was desirable to explore the implications of our results for chaos and ionization in these systems. Paper [4] identified the precession frequency as one of the important parameters of the dynamics, and one expects the onset of chaos when the precession frequency $f_{e}$ is of the order of the Kepler frequency $1 / n^{3}$. For example, for the case of the circular polarization, the transition to chaos occurs at $\mathrm{E}_{0} \sim \omega^{2} l^{2}$. 
Finally, it should be emphasized once again that, in this paper, we review only analytical results on this subject, as has been stated already in the introduction. Papers that used numerical methods to study this problem are beyond the scope of the present mini-review. Nevertheless, for illustrating the accuracy of our analytical results, let us compare them with paper [25] where the authors used numerical methods (finite-element and finite-difference grid methods) for solving the corresponding Schrödinger equation for a hydrogen atom in the field of a circularly polarized high-frequency laser radiation. The authors of paper [25] numerically calculated, in particular, the ground state energy $\mathrm{E}_{0}$ (in atomic units) for several values of some parameter $\mathrm{a}_{0}=(\mathrm{I} / 2)^{1 / 2} / \omega^{2}$, where $\mathrm{I}$ is the laser intensity (all quantities being in atomic units). For example, for the laser frequency $\omega=1.2$ and $\mathrm{a}_{0}=0.3$, from our Equations (20), (22) we obtain $\mathrm{E}_{0}=-0.4652$, while the authors of paper [25] tabulated $\mathrm{E}_{0}=-0.4651$.

Funding: This research received no external funding.

Conflicts of Interest: The authors declare no conflict of interest.

\section{References}

1. Gavrilenko, V.P.; Oks, E.A.; Radchik, A.V. Hydrogen-like atom in the field of high-frequency linearly polarized electromagnetic radiation. Opt. Spectrosc. (USSR) 1985, 59, 411-412.

2. Gavrilenko, V.P. The spectrum of a hydrogen-like atom in high-frequency electromagnetic radiation: Analytic solution. Sov. Phys. JETP 1985, 63, 500.

3. Nadezhdin, B.B.; Oks, E. Physical principles of polarization-spectroscopic measurement of electric and magnetic fields in ionized gases. Sov. Tech. Phys. Lett. 1986, 12, 512.

4. Oks, E.; Davis, J.E.; Uzer, T. Rydberg electron dynamics in high-frequency elliptically polarized microwave fields. J. Phys. B At. Mol. Opt. Phys. 2000, 33, 207. [CrossRef]

5. Ritus, V.I. Shift and splitting of atomic energy levels by the field of an electromagnetic wave. Sov. Phys. JETP 1967, 24, 1041-1044.

6. Zeldovich, Y.B.; Raizer, Y.P. Physics of shock waves and high-temperature hydrodynamic phenomena. Sov. Phys. JETP 1965, 24, 1006.

7. O'Connell, R.F. Ionization energies of hydrogenlike atoms in intense electromagnetic fields. Phys. Rev. A. 1975, 12, 1132. [CrossRef]

8. Savukinas, A.Y. Theoretical consideration of a single-electron atom in an Intense optical field. Litovskiy Fiz. Sbornik 1977, 6, 729-737.

9. Bates, D.R.; Ledsham, K.; Stewart, A.L. Wave functions of the hydrogen molecular ion. Philos. Trans. R. Soc. Ser. A. Math. Phys. Sci. 1953, 246, 215-240. [CrossRef]

10. Vaitekunas, P.P.; Savukinas, A.Y. Determination of the high-frequency dynamic polarizability of atoms by analogy with the two-center problem. Opt. Spectrosc. (USSR) 1983, 54, 17-19.

11. Abramov, D.I.; Slavyanov, S.Y. The two Coulomb centres problem at small inter-centre separations. J. Phys. B At. Mol. Phys. 1978, 11, 2229. [CrossRef]

12. Sholin, G.V. On the nature of the asymmetry of the spectral line profiles of hydrogen in a dense plasma. Opt. Spectrosc. (USSR) 1969, 26, 275.

13. Fok, V.A. Fundamentals of Quantum Mechanics; Mir: Moscow, Russia, 1976; Part 2, Section 5.4.

14. Sobelman, I.I. Introduction to the Theory of Atomic Spectra; Pergamon: Oxford, UK, 1973.

15. Pasternack, S.; Sternheimer, J. An orthogonality property of hydrogenlike radial functions. J. Math. Phys. 1962, 3, 1280. [CrossRef]

16. Bethe, H.A.; Salpeter, E.E. Quantum Mechanics of One- and Two-Electron Atoms; Springer: Berlin, Germany, 1957.

17. Kapitsa, P.L. Dynamic stability of the pendulum when the point of suspension is oscillating. Sov. Phys. JETP $1951,21,588$.

18. Kapitza, P.L. A pendulum with oscillating suspension. Uspekhi Fiz. Nauk. 1951, 44, 7-20. [CrossRef]

19. Landau, L.D.; Lifshitz, E.M. Mechanics; Pergamon: Oxford, UK, 1960; Section 30.

20. Roy, A.E. Orbital Motion; Adam Hilger: Bristol, UK, 1978.

21. Goldstein, H. Classical Mechanics; Addison-Wesley: Reading, MA, USA, 1980.

22. Beletsky, V.V. Essays on the Motion of Celestial Bodies; Birkhäuser/Springer: Basel, Switzerland, 2001. 
23. Oks, E. The Possibility of Measuring Nuclear Shapes by Using Spectral Lines of Muonic Ions. Atoms 2018, 6, 14. [CrossRef]

24. Landau, L.D.; Lifshitz, E.M. Quantum Mechanics; Pergamon: Oxford, UK, 1965.

25. Pont, M.; Gavrila, M. Stabilization of atomic hydrogen in superintense, high-frequency laser fields of circular polarization. Phys. Rev. Lett. 1990, 65, 2362. [CrossRef] [PubMed] 